\title{
BMJ Open Impact of fatigue and insufficient sleep on physician and patient outcomes: a systematic review
}

\author{
Michelle Gates, ${ }^{1}$ Aireen Wingert, ${ }^{1}$ Robin Featherstone, ${ }^{1}$ Charles Samuels, ${ }^{2}$ \\ Christopher Simon, ${ }^{3}$ Michele P Dyson ${ }^{1}$
}

To cite: Gates M, Wingert A, Featherstone $\mathrm{R}$, et al. Impact of fatigue and insufficient sleep on physician and patient outcomes: a systematic review. BMJ Open 2018;8:e021967. doi:10.1136/ bmjopen-2018-021967

- Prepublication history and additional material for this paper are available online. To view these files, please visit the journal online (http://dx.doi. org/10.1136/bmjopen-2018021967).

Received 26 January 2018 Revised 7 August 2018 Accepted 18 August 2018

Check for updates

(c) Author(s) (or their employer(s)) 2018. Re-use permitted under CC BY-NC. No commercial re-use. See rights and permissions. Published by BMJ.

${ }^{1}$ Alberta Research Centre for Health Evidence (ARCHE),

Edmonton Clinic Health

Academy, University of Alberta, Edmonton, Alberta, Canada

${ }^{2}$ Centre for Sleep and Human Performance, Calgary, Alberta, Canada

${ }^{3}$ Canadian Medical Association, Ottawa, Ontario, Canada

Correspondence to Dr Michele P Dyson; mdyson@ualberta.ca

\section{ABSTRACT}

Objectives For physicians in independent practice, we synthesised evidence on the (1) impacts of insufficient sleep and fatigue on health and performance, and patient safety and (2) effectiveness of interventions targeting insufficient sleep and fatigue.

Design We systematically reviewed online literature. After piloting, one reviewer selected studies by title and abstract; full texts were then reviewed in duplicate. One reviewer extracted data; another verified a random $10 \%$ sample. Two reviewers assessed risk of bias. We pooled findings via meta-analysis when appropriate or narratively. Data sources We searched Medline, Embase, PsycINFO CINAHL and PubMed for published studies in April 2016; Medline was updated in November 2017. We searched Embase for conference proceedings, and hand-searched meeting abstracts, association and foundation websites. Eligibility criteria for selecting studies English or French language primary research studies published from 2000 to 2017 examining the effect of fatigue-related or sleep-related exposures or interventions on any outcome among physicians in independent practice and their patients.

Results of 16154 records identified, we included 47 quantitative studies of variable quality. 28 studies showed associations between fatigue or insufficient sleep and physician health and well-being outcomes. 21 studies showed no association with surgical performance, and mixed findings for psychomotor performance, work performance and medical errors. We pooled data from six cohort studies for patient outcomes. For sleep deprived versus non-sleep deprived surgeons, we found no difference in patient mortality $(n=60436$, relative risk (RR) $0.98,95 \% \mathrm{Cl} 0.84$ to $\left.1.15, \mathrm{l}^{2}=0 \%(\mathrm{p}=0.87)\right)$ nor postoperative complications ( $\mathrm{n}=60201$, RR 0.99 , $95 \% \mathrm{Cl} 0.95$ to $\left.1.03, \mathrm{I}^{2}=0 \%(\mathrm{p}=0.45)\right)$. The findings for intraoperative complications and length of stay were considerably heterogeneous.

Conclusions Fatigue and insufficient sleep may be associated with negative physician health outcomes. Current evidence is inadequate to inform practice recommendations.

\section{BACKGROUND}

The working hours of physicians have been a topic of debate for many years. ${ }^{1}$ Beginning in the late 1980s, evidence indicating

\section{Strengths and limitations of this study}

The review was informed by the methods outlined by Cochrane and is reported according to the Preferred Reporting Items for Systematic Reviews and MetaAnalyses (PRISMA) guidelines.

- The review was limited by the quality of the included studies, which was often poor. Confidence in our conclusions may be weakened due to multiple comparisons.

- We have focused on evidence from high-income countries; our findings may not be generalisable to other settings.

that medical resident fatigue could negatively impact their cognitive functioning and performance, resulting in an increased risk of medical error, began to accumulate. ${ }^{2}$ In response, by the early 2000s physicians' regulatory bodies worldwide began to take action toward restricting the work hours of medical residents and ensuring adequate time for recovery between shifts. ${ }^{3-5}$ Since their implementation in the USA by the Accreditation Council for Graduate Medical Education (ACGME), the impact of work hour regulations has been widely researched. Still, evidence for impacts on patient care, resident training and well-being remains equivocal. ${ }^{6-9}$ This is likely because work hours are only one of many contributors to fatigue and physician well-being. In fact, the ACGME has recently reversed the 2011 changes that limited resident work hours to 16 hours per shift and the requirement for 8 hours of time off between shifts. This decision was made in favour of promoting 'flexibility' for residency training programme work hours and scheduling.

The focus on medical trainees has left physicians in independent practice as a relatively neglected group in research and policy. In Canada, there is no concrete regulation on the hours or patterns in which physicians choose to work. ${ }^{10}$ In the absence of clear 
policies, physicians trained under traditional systems may find it difficult to work shorter hours or take more frequent breaks. ${ }^{1}$ Indeed, more than $40 \%$ of practising physicians in the USA work in excess of 80 hours per week. ${ }^{11}$ While long work hours remain a cultural norm in medicine, in comparable high-risk industries (eg, aviation), work patterns and work hours are tightly regulated. ${ }^{12}$ The need for similar evidence-based policies in medicine has become a topic of increased interest. Exemplar of this, an evidence-based guideline for fatigue risk management in emergency medical services,${ }^{13}$ informed by a comprehensive set of systematic reviews, has recently been published. For physicians, it has been argued that there is a need to adapt healthcare systems and provide support in identifying the signs of fatigue and mitigating its risks. ${ }^{1}$

Besides potentially affecting patient outcomes, fatigue can impact the health and well-being of physicians themselves. Burn-out, just one outcome related to fatigue, has been described as epidemic among physicians ${ }^{14-16}$ and ultimately affects recruitment and retention of physicians both in community and acute care settings. While the effect of physician well-being on the sustainability of healthcare systems has recently received increased attention, ${ }^{17}$ evidence-based solutions to burn-out remain relatively elusive. ${ }^{18}$ What is clear is that comprehensive organisational-level efforts are necessary to fully address the issue ${ }^{19}$ Research addressing the factors that influence burn-out and overall physician wellness is needed to inform system-level and individual-level strategies. ${ }^{20} 21$ To date, evidence of the effects of fatigue and the role of chronic insufficient sleep on physicians in independent practice has not been synthesised, making it unclear what gaps in knowledge remain unaddressed.

Given this void, we undertook a systematic review focusing broadly on primary research relevant to the Canadian context as a fundamental starting point to examine the effects of fatigue and chronic insufficient sleep on physicians in independent practice, and on interventions to combat these effects. Our review was guided by the following research questions: Among physicians in independent practice, (1) what are the impacts of fatigue and chronic insufficient sleep on physician health, physician performance and patient safety and (2) what is the effectiveness of interventions that target fatigue and chronic insufficient sleep, in terms of improving physician and patient outcomes?

\section{METHODS}

\section{Review conduct}

The conduct of this systematic review was guided by Cochrane standards. ${ }^{22}$ The research team convened to plan the key research questions and methodology but did not register a formal protocol. The findings are reported in adherence with the Preferred Reporting Items for Systematic reviews and Meta-Analyses (PRISMA) statement. ${ }^{23}$ Ethical approval was not required for this study.
Patient involvement

Patients were not involved.

\section{Literature search}

An information specialist developed a search strategy that included concepts related to physicians, fatigue and sleep. On 13 April 2016, we searched the following online databases with coverage in the biomedical sciences and psychology: Medline, Embase, PsycINFO, CINAHL and PubMed, limited to English and French language articles published from 2000 to 2016. We updated the Medline search in November 2017, as this database offered the highest precision. Though fatigue among physicians is not a new phenomenon, ${ }^{2}$ we limited our search to articles published post-2000 to include studies relevant to current physician practice. Work hour limitations have existed in European countries since 1993, but implementation in the USA $(2003)^{5}$ and Canada (2013) for residents is more recent. ${ }^{24}$ We aimed to include studies published in this era of increased awareness about the potential impacts of long work hours. To locate unpublished studies, we searched Embase for conference proceedings since 2000 and hand-searched meeting abstracts of the Canadian Conference on Physician Health and the International Conference on Physician Health (2012 to 2016). We also searched the following association and foundation websites: American Medical Association, Australian Medical Association, British Medical Association, Canadian Medical Association, European Medical Association, National Sleep Foundation, Ontario Medical Association and the World Medical Association. The complete search strategy undertaken is reported in online supplementary file 1.

\section{Inclusion criteria}

Primary studies (quantitative or qualitative) of fatigue-related or sleep-related exposures or interventions among physicians in independent practice were eligible for inclusion. We included physicians practising in any medical specialty and in any healthcare setting within a high-income country ${ }^{25}$ to identify practices comparable to the Canadian setting. Studies including physicians-in-training were included only if data for physicians in independent practice could be isolated. Exposures of interest included fatigue, insufficient sleep or sleepiness. We also included studies of any intervention that aimed to reduce fatigue or sleep loss with any comparator (or no comparator). All reported outcomes, measured at any time, were eligible for inclusion.

We excluded commentaries, letters, editorials and dissertations. Systematic reviews, health technology assessments, economic evaluations and practice guidelines were excluded, although the reference lists of these as well as the included studies were scanned for potential primary studies. Studies that focused solely on physicians-in-training (eg, trainees, residents, fellows, interns, medical students, junior doctors, registrars) were ineligible. To maintain the focused scope of the review, we 
excluded work hours, work load and any other exposure or intervention that was indirectly related to fatigue or sleep.

\section{Study selection}

The study team piloted the selection criteria, which were then applied by two independent reviewers following a two-phase process. We first screened titles and abstracts for potential relevance. Then, we retrieved all records classified as 'include' or 'unsure' and reviewed their full text for eligibility. Any disagreements between reviewers were resolved by discussion or third-reviewer consultation when necessary.

\section{Data extraction}

Reviewers used a standardised form to extract data in Microsoft Office Excel (V.2016; Microsoft, Redmond, Washington, USA). One reviewer independently extracted data from each included study and a second reviewer verified a random $10 \%$ sample. Since no major errors or omissions were noted, we did not undertake further verification.

We extracted the following data: country of publication, funding source, study design, inclusion and exclusion criteria, population characteristics (ie, sample size, age and gender distribution, physician specialty), setting (ie, physician workplace, urban or rural), exposure or intervention, definition of fatigue or insufficient sleep, sleep and fatigue scales used and timing of measurement, comparators (if applicable) and outcomes.

\section{Risk of bias appraisal}

Two reviewers independently assessed the risk of bias in each included study using standard tools. Disagreements were resolved via discussion or by consulting a third reviewer. We used the Cochrane Risk of Bias tool ${ }^{22}$ to assess randomised controlled trials (RCTs). Adapted versions of the tool developed by the Effective Practice and Organization of Care group ${ }^{26}$ were used to assess before-after and time series studies. We used the Newcastle-Ottawa Quality Assessment Scale ${ }^{27}$ to appraise cohort studies. We adapted the scale to assess cross-sectional studies and the one non-comparative study.

\section{Evidence synthesis}

We considered clinical and methodological heterogeneity in our decision on whether to proceed with meta-analysis for the outcomes identified. For most outcomes, we found high levels of heterogeneity in study design, populations, exposures or interventions, and outcome measures and chose not to pool the data via meta-analysis. Thus, we have presented the findings for most outcomes narratively and in summary tables.

When statistical pooling was appropriate, this was undertaken using Review Manager (RevMan V.5.3, Copenhagen: The Nordic Cochrane Centre, the Cochrane Collaboration, 2014) via pairwise meta-analysis using the DerSimonian and Laird random effects model (given expected heterogeneity). ${ }^{28}$ We pooled dichotomous outcomes using the relative risk ( $\mathrm{RR}, 95 \% \mathrm{CI})$ and continuous outcomes using the mean difference $(95 \% \mathrm{CI})$ since the units across studies were consistent (eg, minutes). When meta-analysis was conducted, we assessed statistical heterogeneity using the $\chi^{2}$ test (using $\mathrm{p}=0.10$ as the threshold for significance) and quantified the extent of heterogeneity using the $\mathrm{I}^{2}$ statistic. ${ }^{29}$ We considered an $\mathrm{I}^{2}$ value of $0 \%$ to $40 \%$ to be low (potentially unimportant), $30 \%$ to $60 \%$ to be moderate, $50 \%$ to $90 \%$ to be substantial and $75 \%$ to $100 \%$ to be considerable heterogeneity. ${ }^{22}$ Subgroup and sensitivity analyses were conducted when appropriate to explore heterogeneity. We intended to assess small study bias visually by inspecting funnel plots and statistically using Egger's regression test, but did not due to the small number (ie, less than 8 ) of studies included in the meta-analyses. ${ }^{30}$

When data were not presented in the format required for meta-analysis, we estimated means or SDs using standard equations. We used the median instead of the mean for one study ${ }^{31}$ for the outcomes of length of stay and operating time. Additionally, for one study ${ }^{32}$ in the length of stay analysis where the SD could not be estimated, we substituted the mean variance of other studies within the meta-analysis. ${ }^{33}$

\section{RESULTS}

We identified 16083 unique records via the database searches, 56 grey literature sources and 15 additional records in reference lists of systematic reviews. We excluded 15016 citations by title and abstract, and another 1091 by full text. Forty-seven studies ${ }^{313234-78}$ were eligible for inclusion, and six 313241586377 were included in meta-analysis for the outcomes of operating time, intraoperative and postoperative complications, patient mortality and length of hospital stay. Figure 1 shows the flow of studies through the selection process.

\section{Included study characteristics}

A summary of the study characteristics is provided in table 1 . Online supplementary file 2 presents descriptive information for each included study. There were 45 observational studies ${ }^{31} 32$ 34-39 41-74 76-78 and 2 intervention studies. ${ }^{40} 75$ All studies were quantitative. Nearly half $(n=20 / 47,43 \%)$ of the studies took place in North America $313235373841454857-606263$ 65-68 7277 and slightly more than one-third $(\mathrm{n}=16 / 47,34 \%)$ in Europe. ${ }^{3436394042464750-536164737576}$

The 47 studies reported outcomes for 36190 (range $=6$ to 7905$)$ physicians and 69809 (range $=270$ to 38 978) adult patients. About half reported on surgeons $(n=13 / 47,28 \%),{ }^{31} 323441454854586263667577$ or anesthesiologists/physician anaesthetists $(n=10 / 47$, $21 \%) .363739434450-525960$ Where it was reported, the samples tended to be predominantly male $(\mathrm{n}=30 / 38,79 \%)$ and physician age varied widely. Hospitals were the most common setting $(n=37 / 47$, 79\%). ${ }^{313234-3739-4345-4749-525457-6163-7073-757778}$ Inthestudieswhereitwas 


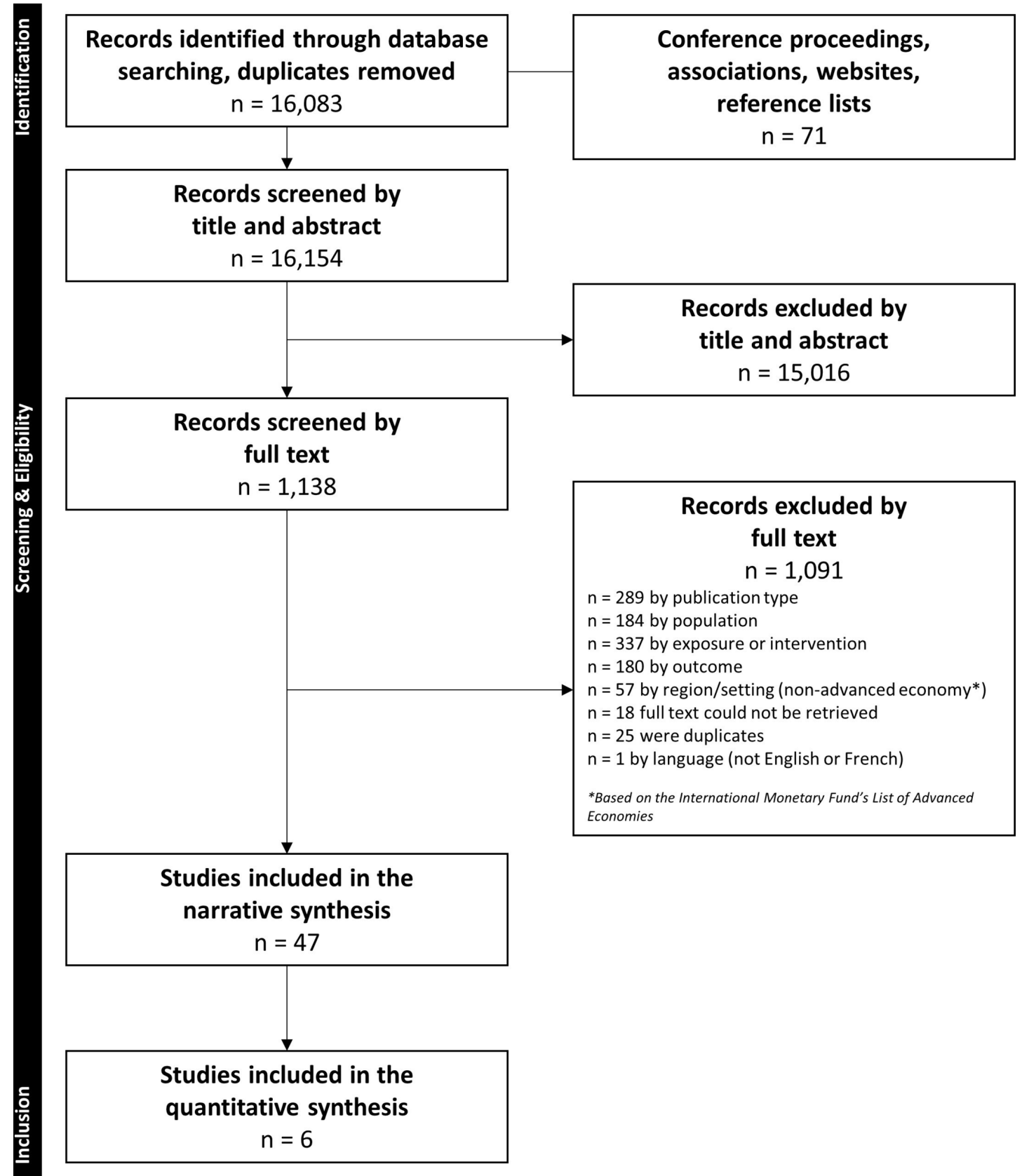

Figure 1 Flow of records through the selection process.

reported $(n=16 / 47,34 \%), 31323438404143455051555658657677$ all but four studies ${ }^{31555677}$ took place in solely an urban setting.

Fifteen (32\%) studies reported on fatigue-related exposures (eg, as a source of stress, exhaustion, physical fatigue; hereafter referred to as 'fatigue'), 35 $4045485763-7173$ while others $(n=37 / 47,79 \%)$ reported on sleep-related exposures (eg, sleep hours, insufficient sleep, sleep deprivation, sleep disruption, sleepiness; hereafter referred to as 'insufficientsleep'). ${ }^{31323436-4749-5658-626467717274-78}$ Afew ( $\mathrm{n}=5 / 47$, $11 \%)$ reported on both. ${ }^{4045646771}$ In some cases $(n=18 / 47$, $38 \%$ ), fatigue or insufficient sleep were related to overnight work or long on-call shifts. $31323437404143454650515358-60637577$ Measured outcomes varied widely and were ultimately organised into physician physical and mental health, physician performance and risk of error, and patient outcomes.

\section{Risk of bias appraisal}

The overall quality of the body of research was poor; $62 \%(n=29 / 47)$ of studies were rated at unclear or high risk of bias. Of the two RCTs, one was rated as unclear overall risk of bias ${ }^{75}$ and one as high risk. ${ }^{40}$ All cohort studies were at low risk of bias (mean score: $8.4 / 9$, range: 8-9)..$^{313241586377}$ All of the before-after studies were rated as high risk of bias. ${ }^{34} 450$ The single time series study was assessed at high risk of bias. ${ }^{51}$ The cross-sectional studies varied in performance (mean score: $3.0 / 5$, range: $1-4)$; only one-third $(n=12 / 34,35 \%)$ were at low risk of bias. ${ }^{39} 4244475259606970727476$ The one non-comparative 
Table 1 Summary characteristics of the included studies

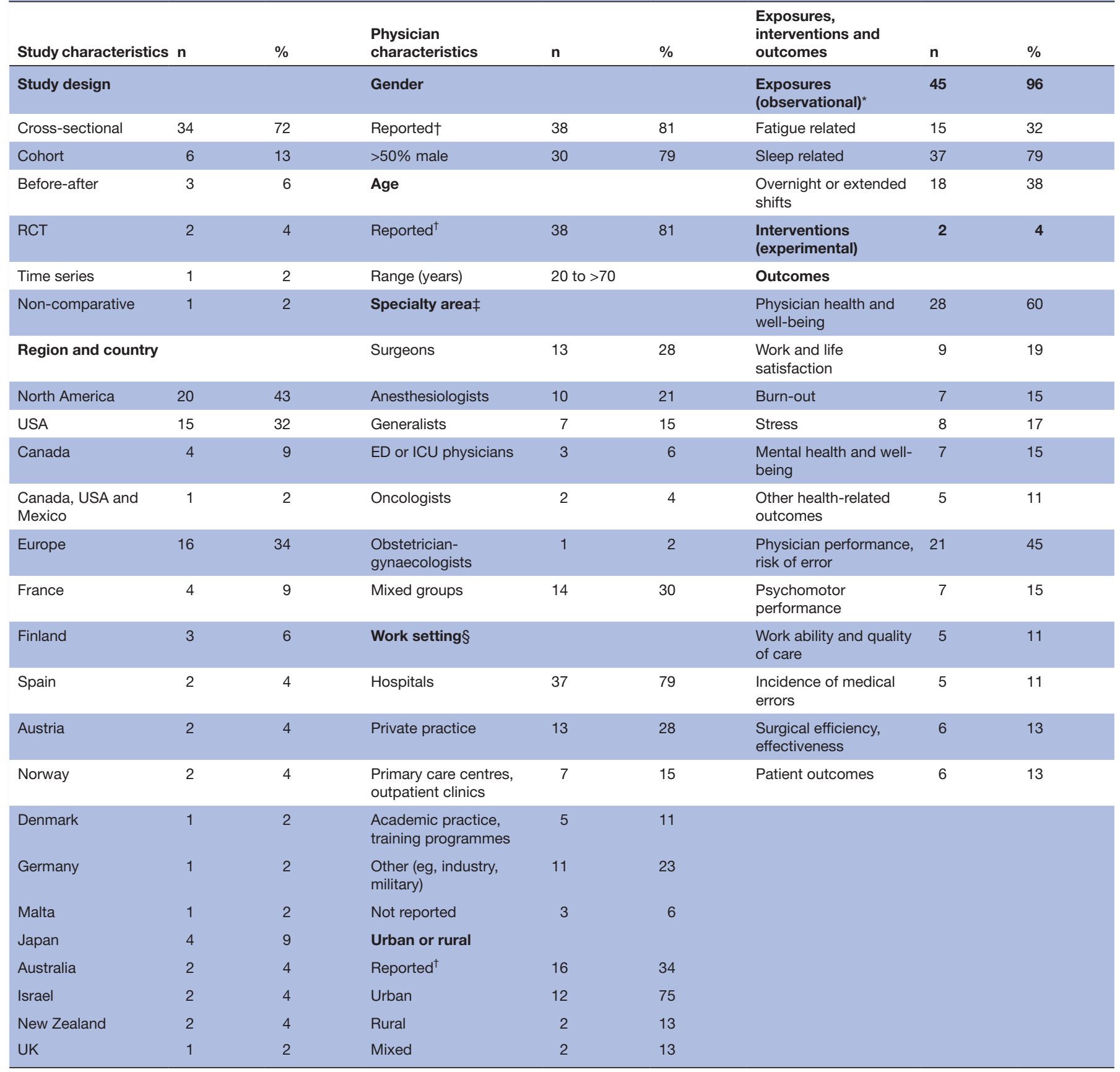

*Exposures that have been directly related to an outcome. Some studies included multiple exposures.

†Percentages presented using the total number of studies where the outcome was reported as the denominator.

$\ddagger$ Anesthesiologists include physician anaesthetists; generalists include primary care physicians, internists and general practitioners; mixed groups refers to studies including more than one physician group or specialty (usually large-scale surveys). In some studies, multiple distinct groups were represented.

$\S$ As defined by the authors. Values for the settings will exceed $100 \%$ because studies may occur in more than one setting.

ED, emergency department; ICU, intensive care unit; RCT, randomised controlled trial.

study was at unclear risk of bias. ${ }^{43}$ Detailed assessments of the sources of bias per study are shown in online supplementary file 3 .

\section{Physician health and well-being outcomes}

Twenty-eight studies reported on physician health and well-being-related outcomes, ${ }^{35} 36 \quad 38-4042 \quad 46-48 \quad 50-57606264 \quad 6768 \quad 70-72 \quad 7476 \quad 78$ including burn-out $(n=7)$, stress $(n=8)$, mental health and well-being $(n=7)$, life and job satisfaction $(n=9)$ and other markers of health $(n=5)$ (online supplementary file 4).

Seven cross-sectional studies reported on burn-out (five low, ${ }^{39} 70727476$ one unclear, ${ }^{54}$ one high risk of bias ${ }^{62}$ ) among surgeons, ${ }^{54}{ }^{62}$ anesthesiologists, ${ }^{39}$ generalists ${ }^{76}$ and other mixed groups. ${ }^{70} 7274$ Two studies reported on surgeons; the larger ( $\mathrm{n}=2$ 564, low risk of bias) study of 
neurosurgeons showed increased odds of burn-out with sleep deprivation (hours of sleep per night; OR 0.84, $95 \%$ CI 0.75 to $0.94, \mathrm{p}=0.002) .{ }^{54}$ Among anesthesiologists, one study $(n=565$, low risk of bias) indicated that burn-out (measured via Maslach Burnout Inventory) was more prevalent among the sleep-deprived ('lack of sleep' on one question; $47.6 \%$ vs $16.3 \%, \mathrm{p}<0.001) .{ }^{39}$ In one small $(n=11)$ study of generalists, those with burn-out (measured via Pines Burnout Measure) had poorer Pittsburgh Sleep Quality Index scores (7.24 \pm 4.17 vs. $2.72 \pm 2.22, \mathrm{p}<0.001) .{ }^{76}$ In the two larger studies of mixed physician groups (low risk of bias), burnout (measured via 5-point scale) was more prevalent among those who were sleep deprived ( $<7$ hours of sleep per 24hours; $39.6 \%$ vs $26.4 \%, \mathrm{p}<0.05),{ }^{72}$ and physical fatigue ('feeling tired' on a 7-point scale) was correlated with burn-out (Shirom-Melamed Burnout Measure; $r=0.88, \mathrm{p}<0.05) .^{70}$ In summary, evidence from seven cross-sectional studies ( $71 \%$ at low risk of bias) showed associations between insufficient sleep and burn-out.

Six cross-sectional studies (two low, ${ }^{47}$ one unclear, ${ }^{46}$ three high risk of bias ${ }^{356264}$ ), one uncontrolled beforeafter study (high risk of bias ${ }^{50}$ ) and one intervention study (high risk of bias ${ }^{40}$ ) reported on stress outcomes among surgeons, ${ }^{62}$ anesthesiologists, ${ }^{50}{ }^{52}$ emergency physicians, ${ }^{4064}$ internal medicine physicians ${ }^{46}$ and mixed groups. $^{3547}$ In a small sample $(n=20)$ of internal medicine physicians, insufficient sleep related to a 24-hour call shift showed no association with biochemical or physiological stress parameters, except levels of thyroid-stimulating hormone, which was higher post-shift $(\mathrm{p}=0.049$, data not reported). ${ }^{46}$ The remaining observational studies suggested that there was an association between insufficient sleep or fatigue and stress. The one study of orthopaedic surgeons $(n=264$, high risk of bias) showed that insufficient sleep (measured on a 3-point scale) and psychological distress (measured via General Health Questionnaire-12) were correlated (data not reported, $\mathrm{p}<0.001){ }^{62}$ The two reports on anesthesiologists were of varied quality; the larger $(n=328$, low risk of bias) study showed that stress symptoms (measured via Modified Occupational Stress Questionnaire) were predicted by sleep sufficiency (self-reported on one question, $\beta=-0.269, \mathrm{p}<0.001) .^{52}$ Among the two studies reporting on mixed groups of physicians, the larger $(n=1541$, low risk of bias) study showed an association between sleep problems (four questions derived from Jenkins scale) and psychological distress (General Health Questionnaire-12; $\beta=0.18, p<0.001) .{ }^{47}$ One RCT assessed the impact of insufficient sleep from shift work (14-hour or 24-hour shifts), showing that stress (on a Visual Analogue Scale) among emergency physicians $(n=17)$ was higher following the shift as compared with a control day (data not reported, $\mathrm{p}<0.05) .{ }^{40}$ In summary, evidence from one intervention study at high risk of bias and all but one of the seven observational studies (29\% at low risk of bias) that we identified supported an inverse association between fatigue or sleep deprivation and stress.
Seven cross-sectional studies (two low, ${ }^{52}{ }^{60}$ three unclear, ${ }^{67} 71{ }^{78}$ two high risk of bias ${ }^{36}$ ) reported on aspects of mental health including addiction or substance misuse, ${ }^{36} 5371$ depression, ${ }^{78}$ thoughts of suicide, ${ }^{52}$ mood disturbance $^{60} 71$ and overall well-being. ${ }^{67}$ One study, ${ }^{53}$ which was at high risk of bias, showed no association between hours of sleep when on call and hazardous drinking behaviours (via Alcohol Use Disorder Identification Test). Meanwhile, the six other studies all showed associations between insufficient sleep and fatigue and reduced mental health. Three studies reported on anaesthetists, ${ }^{36} 5260$ with two large surveys showing increased odds of tobacco (OR 1.42, 95\% CI 1.04 to 1.94) and tranquillizer/hypnotics (OR 3.26, 95\% CI 2.12 to 5.02) dependency being predicted by sleep deprivation (measured by one question), ${ }^{36}$ and sleep disturbance being associated with thoughts of suicide (using a 4-point scale; $\mathrm{p}=0.009) .^{52}$ A small study $(n=21)$ showed greater mood disturbance following a 17-hour night shift than a usual day (Profile of

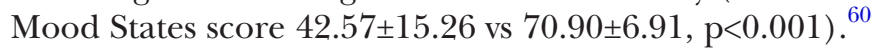
Among oncologists $(n=241)$, overall well-being was predicted by lower levels of fatigue after controlling for personal and professional characteristics (assessed via Linear Analogue Scale quality of life survey, $\mathrm{p}=0.002) .{ }^{67}$ A large $(n=3$ 862, unclear risk of bias) study of physicians showed that insufficient sleep (lower sleep hours when not at work in the past month) was associated with increased odds of depression (Quick Inventory Depressive Scale; OR 2.70, 95\% CI 1.82 to 4.03 for men; OR 2.38, $95 \%$ CI 1.11 to 5.10 for women). ${ }^{78}$ In open-ended questions, senior physicians in one study (unclear risk of bias) attributed the development of mental illness to tiredness and stress at work. ${ }^{71}$ In summary, seven cross-sectional studies (29\% at low risk of bias) were identified, and of these six supported an association between insufficient sleep or fatigue and negative mental health outcomes.

Nine cross-sectional studies (four low, ${ }^{42} 47 \quad 7274$ two unclear, ${ }^{55}$ three high risk of bias ${ }^{38} 4862$ ) reported on outcomes related to job satisfaction, ${ }^{424748557274}$ life satisfaction $^{386272}$ or work-life balance. ${ }^{6872}$ The six studies that investigated job satisfaction were all at low risk of bias and generally included mixed groups of physicians ${ }^{47} 7274$; one study reported on general practitioners, ${ }^{55}$ another on surgeons ${ }^{48}$ and one on mixed specialties. ${ }^{42}$ Three studies showed that reductions in sleep duration and/or quality ${ }^{47874}$ were associated with reduced job satisfaction. Meanwhile, one showed no association between insufficient sleep ( $<7$ hours per 24-hour period) and career satisfaction (measured on a 5-point Likert scale), ${ }^{72}$ and another showed no relationship between earlier sleep disturbance (Jenkins Scale) and later job demands or job control (measured via 5-point scale). ${ }^{42}$ A single study $(n=92)$ reporting on rural general practitioners indicated that frequent sleep disturbance (measured on a 7-point scale) predicted the intention to retire early (OR 2.91, $95 \%$ CI 1.11 to $7.6, \mathrm{p}<0.05) .{ }^{55}$ In summary, six cross-sectional studies (all at low risk of bias) were identified, and all but two ${ }^{42} 72$ of these studies showed that insufficient 
sleep and fatigue were associated with reductions in satisfaction.

Three studies reported on life satisfaction. ${ }^{38} 6272$ Of two studies among mixed physician groups, ${ }^{38} 72$ the one larger $(n=840)$ study showed that insufficient sleep $(<7$ hours per day) was a predictor of reduced life satisfaction (measured on a 5-point Likert scale; OR 0.44, $95 \%$ CI 0.29 to $0.67, \mathrm{p} \leq 0.05) .{ }^{72}$ One study at high risk of bias reported on orthopaedic surgeons $(n=264)$, showing that sleep deprivation (measured via 3-point scale) was correlated with lower marital satisfaction (Revised Dyadic Adjustment Scale; data not reported, $\mathrm{p}<0.001) .{ }^{62}$ Two large studies at low or unclear risk of bias reported on work-life balance. ${ }^{68}{ }^{72}$ Among oncologists ( $n=1$ 117), reduced satisfaction with work-life balance (measured on a 5-point Likert scale) was predicted by high levels of fatigue (measured via 10-point Visual Analogue Scale), even when controlling for personal and work-related factors and burn-out (OR $0.489,95 \%$ CI 0.337 to 0.710 , $\mathrm{p}<0.001) .{ }^{68}$ Among a mixed group of physicians $(\mathrm{n}=840$, low risk of bias), insufficient sleep ( $<7$ hours in a typical 24-hour period) predicted a reduced perception of having balanced personal and professional commitments (5-point Likert scale; OR $0.46,95 \%$ CI 0.31 to 0.71 , $\mathrm{p} \leq 0.05$ ) ${ }^{72}$ In summary, three cross-sectional studies (all unclear or high risk of bias) supported an association between insufficient sleep or fatigue and reduced life satisfaction, and two cross-sectional studies $(50 \%$ low risk of bias) supported an association with reduced work-life balance.

Four cross-sectional studies (three unclear, ${ }^{56} 57$ one high risk of bias $^{38}$ ) and one time series study (high risk of bias $^{51}$ ) reported on other health-related outcomes. Among a mixed group of physicians $(\mathrm{n}=180)$, one study at high risk of bias showed that Epworth Sleepiness Scale scores were higher among physicians who worried about having a car accident while driving home (7.0 vs 5.4, $\mathrm{p}<0.001) .{ }^{38}$ Among generalists $(\mathrm{n}=578)$, almost 1 in $10(8.7 \%)$ admitted to falling asleep while driving due to fatigue. ${ }^{57}$ Also among generalists $(n=92)$, those with frequent work-related sleep disturbance (measured on a 7-point scale) were at increased odds of sickness presenteeism (OR 2.92, 95\% CI 1.19 to $7.16, p=0.02) .{ }^{56}$ The one time series study concluded that a single 24-hour shift did not cause major chronodisruption (based on serum melatonin measurement) among anaesthetists $(\mathrm{n}=10) .{ }^{51}$ Meanwhile, open-ended comments from a large sample $(n=3550)$ of senior physicians suggests that they attributed the development of physical health problems to a lifestyle of insufficient sleep, poor eating habits and lack of exercise imposed by their jobs. ${ }^{71}$ In summary, five cross-sectional studies ( $0 \%$ at low risk of bias) supported associations between insufficient sleep and fatigue and varied deleterious health outcomes (ie, car accidents, sickness presenteeism, physical health problems). One time series study at high risk of bias did not support such a relationship.

\section{Physician performance and risk of errors}

Twenty-onestudies reported on physician performanceand safety-related outcomes, ${ }^{31323437384143-4749505961656669717375}$ includingsurgicalefficiencyandeffectiveness ( $\mathrm{n}=6$ ), psychomotor performance $(n=7)$, work ability and quality of care $(n=5)$ and medical errors $(n=5)$ (online supplementary file 4).

Four cohort studies (all low risk of bias ${ }^{31} 324163$ ), one before-after study (high risk of bias $^{34}$ ) and one RCT (high risk of bias ${ }^{75}$ ) examined the effects of insufficient sleep from overnight work or extended shifts, during surgeries $^{31} 3241$ or laparoscopic simulations. ${ }^{3475}$ We pooled the data from these studies 31324163 via meta-analysis, which showed no difference in operating time (sometimes referred to as surgeon efficiency) between sleep deprived and non-sleep deprived surgeons (figure 2; $\mathrm{n}=50046$, mean difference (MD) $-0.14,95 \%$ CI -1.60 to $\left.1.33, \mathrm{I}^{2}=0 \%(\mathrm{p}=0.70)\right)$. Of studies not meta-analysed, the small ( $n=29)$ before-after study showed no impact of sleep deprivation from shift work nor of sleep hours on performance on a laparoscopic simulation (LapSimGyn). ${ }^{34}$ One small $(n=64)$ intervention study compared a 24-hour shift to a usual work day, also finding no detriment to performance on a laparoscopic simulation (minimally invasive surgical trainer-virtual reality) despite diminished sleep hours while working on-call. ${ }^{75}$ In summary, pooled data from four cohort studies (100\% low risk of bias) showed no effect of insufficient sleep on surgical efficiency. Additional data from one RCT (high risk of bias) and one before-after study (high risk of bias) also showed no association between insufficient sleep and performance on laparoscopic simulations.

Two before-after studies (high risk of bias ${ }^{450}$ ) and five cross-sectional studies (two low, ${ }^{49}{ }^{59}$ two unclear, ${ }^{37}{ }^{46}$ one high risk of bias $^{61}$ ) reported on psychomotor performance outcomes among surgeons, ${ }^{45}$ anesthesiologists, ${ }^{37435059}$ emergency physicians ${ }^{61}$ and internal medicine physicians. ${ }^{46}$ Among a small group of surgeons $(n=9)$,

\begin{tabular}{|c|c|c|c|c|c|c|c|c|c|c|c|}
\hline \multirow[b]{2}{*}{ Study or Subgroup } & \multicolumn{3}{|c|}{ Sleep deprived } & \multicolumn{3}{|c|}{ Not sleep deprived } & \multicolumn{3}{|c|}{ Mean Difference } & \multirow{2}{*}{\multicolumn{2}{|c|}{$\begin{array}{c}\text { Mean Difference } \\
\text { IV, Random, } 95 \% \mathrm{Cl}\end{array}$}} \\
\hline & Mean & SD & Total & Mean & SD & Total & Weight & IV, Random, $95 \% \mathrm{Cl}$ & Year & & \\
\hline Ellman 2004 & 107.7 & 55.24 & 339 & 107.4 & 56.05 & 6412 & $5.9 \%$ & $0.30[-5.74,6.34]$ & 2004 & + & \\
\hline Schieman 2008 & 114 & 226.6 & 22 & 148 & 226.6 & 248 & $0.0 \%$ & $-34.00[-132.80,64.80]$ & 2008 & & \\
\hline Chu 2011 & 110.2 & 119.13 & 1678 & 114 & 120.88 & 2369 & $3.8 \%$ & $-3.80[-11.30,3.70]$ & 2011 & & \\
\hline Govindarajan 2015 & 156 & 75.56 & 19489 & 156 & 80 & 19489 & $90.2 \%$ & $0.00[-1.54,1.54]$ & 2015 & & \\
\hline Total $(95 \% \mathrm{Cl})$ & & & 21528 & & & 28518 & $100.0 \%$ & $-0.14[-1.60,1.33]$ & & & \\
\hline \multicolumn{10}{|c|}{$\begin{array}{l}\text { Heterogeneity: } \text { Tau }^{2}=0.00 ; \mathrm{Chi}^{2}=1.42, \mathrm{df}=3(\mathrm{P}=0.70) ; \mathrm{I}^{2}=0 \% \\
\text { Test for overall effect: } Z=0.18(P=0.86)\end{array}$} & $\begin{array}{cccc}-100 & -50 & 0 & 50 \\
\text { Favors sleep deprived } & \text { Favors non-s }\end{array}$ & $\begin{array}{l}100 \\
\text { eprive }\end{array}$ \\
\hline
\end{tabular}

Figure 2 Forest plot for operating time among sleep deprived and non-sleep deprived surgeons. 
performance on a virtual ring transfer task deteriorated after an on-call shift (data not reported, $\mathrm{p}<0.05) .{ }^{45}$ The four studies among anaesthetists reported mixed findings. One small $(\mathrm{n}=11)$ before-after study showed longer reaction times $(690.8 \pm 73.4$ vs $746.5 \pm 113.7 \mathrm{~ms})$ and reduced concentration ability ( $26.4 \pm 23.5$ vs $56.3 \pm 23.0$ on a 100-point scale, $\mathrm{p}=0.007$ ) following a 24-hours shift with insufficient sleep ${ }^{50}$; two others found that insufficient sleep due to overnight shifts was associated with slower reaction times. ${ }^{435}$ Conversely, a small study $(n=11)$ found no effect of overnight shift work with insufficient sleep on any measure of psychomotor performance except Hopkin's Verbal Learning Test (t-score of $48.6 \pm 7.6$ vs $41.5 \pm 9.9, \mathrm{p}=0.04) .{ }^{37}$ Among emergency physicians $(\mathrm{n}=18)$, one study (high risk of bias) showed that those who were sleep deprived ( $<5$ hours sleep after a 24-hour shift) had a reduced performance on most but not all psychomotor tests (Battery Test Reaction 5), ${ }^{61}$ while among internal medicine physicians $(\mathrm{n}=20$, low risk of bias), neurocognitive parameters did not seem to worsen post-call. ${ }^{46}$ In summary, two before-after ( $0 \%$ low risk of bias) and five cross-sectional studies ( $40 \%$ low risk of bias) showed mixed results for the association between fatigue or insufficient sleep and psychomotor performance.

Five cross-sectional studies (two low, ${ }^{47}{ }^{69}$ one unclear, ${ }^{71}$ two high risk of bias ${ }^{38}{ }^{65}$ ) reported on associations between sleep deprivation or fatigue and work ability or perceived performance, all among mixed groups of physicians. ${ }^{3847656971}$ The two large studies at low risk of bias showed that sleep problems and fatigue were inversely associated with physicians' perceived quality of work. ${ }^{47}{ }^{69}$ Among 1541 physicians in Finland, sleeping problems (measured by four questions from the Jenkins Scale) were inversely associated with scores on the Work Ability Index $(\beta=-0.29, \mathrm{p}<0.001),{ }^{47}$ while a study of 890 physicians from Israel demonstrated that perceived quality of care was predicted by fatigue (1 item on the Shirom-Melamed Burnout Measure) even after controlling for components of burn-out $(\beta=0.17$, $\mathrm{p}<0.05) .{ }^{69}$ Similarly, in one study, comments from senior physicians suggested that continual tiredness and exhaustion negatively affected their perceived competence. ${ }^{71}$ The two studies ${ }^{38} 65$ that were at high risk of bias had conflicting findings. In summary, five cross-sectional studies ( $40 \%$ at low risk of bias) reported on perceived work performance; those that were at low risk of bias supported an association between fatigue or insufficient sleep and reduced performance.

Five cross-sectional studies (one low, ${ }^{44}$ two unclear, ${ }^{4966}$ two high risk of bias ${ }^{38}$ ) reported on associations between insufficient sleep or fatigue and self-reported medical errors among surgeons, ${ }^{66}$ anesthesiologists ${ }^{44}$ and mixed groups of physicians. ${ }^{38} 4973$ A large $(n=7$ 905) study at unclear risk of bias showed that only $6.9 \%$ of surgeons reported fatigue as the most important contributor to medical errors. ${ }^{66}$ Among anesthesiologists, a smaller study $(n=183)$ at low risk of bias showed that the risk of self-reported fatigue-related errors increased with more nights of work-related sleep disturbance (RR 1.25, 95\% CI 1.06 to 1.49$).{ }^{44}$ Two of the studies reporting on mixed groups of physicians had conflicting results, ${ }^{38} 49$ while another reported that physicians' opinions on the association between fatigue and prescribing errors differed by work setting. ${ }^{73}$ One-third $(34 \%)$ of community-based, $96 \%$ of hospital-based and $8 \%$ of office-based physicians believed that there was a high or very high association between fatigue and prescribing errors $(p<0.05) .{ }^{73}$ In summary, five cross-sectional studies (20\% at low risk of bias) reported on self-reported errors and these showed mixed findings for associations with fatigue or insufficient sleep.

\section{Patient outcomes}

Six large ( $\mathrm{n}=270$ to 38 978) cohort studies at low risk of bias reported on patient outcomes, all related to surgical $^{31} 3241586377$ or obstetric $^{58}$ procedures (online supplementary file 4). In these studies, insufficient sleep or fatigue were typically defined as overnight work prior to a daytime procedure, ${ }^{3141586377}$ though two studies measured sleep hours ${ }^{32}$ or 'sleep opportunity'. We pooled data for procedures performed by sleep deprived versus non-sleep deprived surgeons (or obstetrician-gynaecologists in one $\operatorname{case}^{58}$ ). Analyses showed no difference in the rate of postoperative complications (figure 3; five studies, ${ }^{31} 32416377 \mathrm{n}=60201$, RR 0.99 , $95 \%$ CI 0.95 to $1.03, \mathrm{I}^{2}=0 \%(\mathrm{p}=0.45)$ nor patient mortality

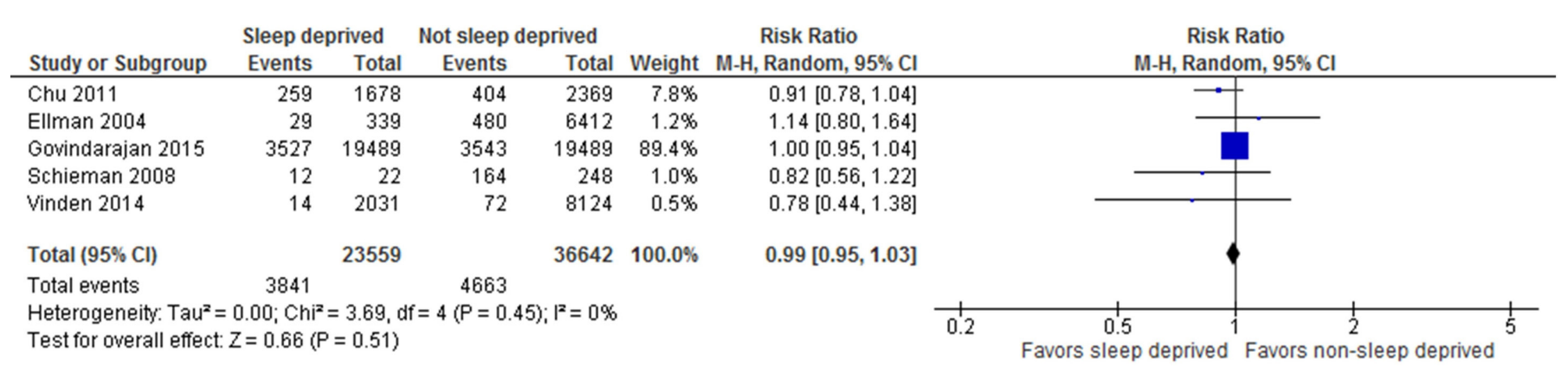

Figure 3 Forest plot for postoperative complications among surgeries performed by sleep deprived and non-sleep deprived surgeons. Vinden et $a l^{77}$ reported iatrogenic injuries, Schieman et al ${ }^{63}$, Govindarajan et al ${ }^{31}$ and Chu et al ${ }^{32}$ reported postoperative complication rate and Ellman ${ }^{41}$ reported postoperative complications (other types of complications reported not included in the analysis). 


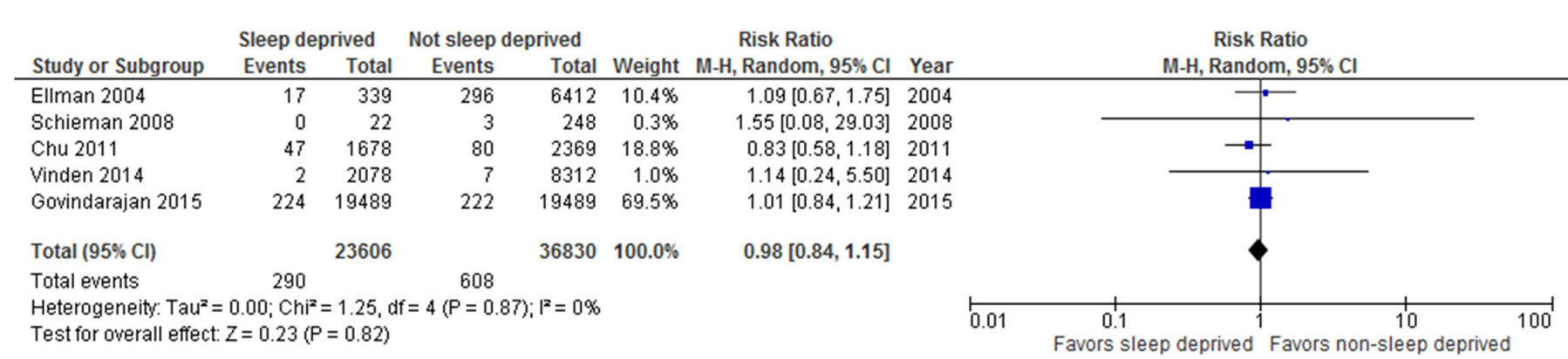

Figure 4 Forest plot for patient mortality among surgeries performed by sleep deprived and non-sleep deprived surgeons.

(figure 4; five studies, ${ }^{31} 32416377 \mathrm{n}=60436$, RR $0.98,95 \%$ CI 0.84 to $\left.1.15, \mathrm{I}^{2}=0 \%(\mathrm{p}=0.87)\right)$. One study ${ }^{77}$ in the mortality analysis reported the number of deaths only as $\leq 5$. We assumed two events for this study (midpoint between 0 and 5); sensitivity analysis using the lowest (ie, 0) and highest (ie, 5) possible number of events did not change the overall result (online supplementary file 5). We found considerable between-study heterogeneity in the analyses for intraoperative complications $\left(\mathrm{I}^{2}=82 \%\right)$ and length of stay $\left(\mathrm{I}^{2}=86 \%\right)$, which could not be explained via subgroup analyses by procedure type, thus we have suppressed the average estimates of effect. For length of stay, the results of one study on cardiac surgeries favoured sleep deprived surgeons, ${ }^{32}$ while the others ${ }^{314163}$ had null results. For intraoperative complications, the findings of one study ${ }^{63}$ favoured non-sleep deprived surgeons, but the others ${ }^{58} 77$ had null results.

\section{DISCUSSION}

Fatigue and chronic insufficient sleep are two potential drivers of reduced physician well-being ${ }^{17}$ that have thus far been understudied in physicians in independent practice. Burn-out is becoming increasingly prevalent among physicians, ${ }^{14-16}$ and recent research indicates that comprehensive individual-level and system-level strategies are needed to address the problem..$^{6-91921}$ We have systematically reviewed evidence from a heterogeneous array of available studies reporting on diverse outcomes related to physicians in independent practice and their patients. The included studies were often at high or unclear risk of bias, included small samples of physicians and inconsistently measured and reported exposures and outcomes. The key message gleaned from this review is that despite growing interest in the topic of physician wellness, the robust evidence needed to inform individual-level and systems-level fatigue management strategies is lacking.

Traditionally, much of the fatigue-related research has focused on hazards to patients. The current review included six cohort studies showing that insufficient sleep and/or fatigue did not seem to result in increased rates of patient morality or postoperative complications; findings for length of stay and intraoperative complications were inconclusive. Evidence for psychomotor performance, surgical skills and errors suggest that there is indeed a potential for negative outcomes. The included studies, like many of the others in this and other systematic reviews, ${ }^{79}$ employed indirect definitions that make it difficult to classify sleep deprived physicians with certainty. In recent years, there has been a shift away from the singular focus on patient safety toward a more comprehensive view that also considers the detrimental effects of fatigue, sleep loss and other occupational hazards on physician wellness. ${ }^{80}$ Evidence from this review supports that fatigue and insufficient sleep may be negatively associated with physician health and well-being. It is now recognised that health systems cannot be sustained by a workforce that is facing an epidemic of burn-out. ${ }^{198182}$

In light of high rates of burn-out, the ongoing dialogue about the need for a cultural shift in the practice of medicine $^{8384}$ is now more important than ever. Recognition of the potential effects of physician fatigue on patients, physicians and healthcare systems as a whole must be emphasised at a systemic level, encouraging a shift in which the risks are viewed as unacceptable. ${ }^{2080}$ Likewise, although research to date has focused largely on individual-level approaches to address burn-out, it is now clear that placing the burden of a system-level problem solely on the individual is unlikely to bring about significant and lasting change. ${ }^{85}$ Recent research has highlighted physician burn-out as a system-driven issue that will require corresponding national-scale multicomponent solutions. ${ }^{1} 198182$ As such, in the past several years both the American and Canadian Medical Associations have developed policies and programmes that address physician health. ${ }^{81} 86$ The Canadian Medical Association's new policy on physician health calls on broad stakeholder groups (eg, policy-makers, regional health authorities, governments) to take shared responsibility for the health of physicians and to make meaningful and concerted efforts towards promoting a healthy and sustainable workforce. $^{81}$

The most salient finding of this review is that the current evidence is insufficient to inform policy and practice. Correspondingly, a 2016 research summit on physician wellness and burn-out outlined the need for timely, relevant and methodologically robust research to inform practice and policy. ${ }^{21}$ The findings herein may be used as motivation for researchers and practitioners to develop and design methodologically strong research programmes related to physician fatigue, inform successful research 
grant proposals, and lobby healthcare organisations to increase the focus on physician fatigue management programmes. It will be important to make use of existing validated measures ${ }^{87-89}$ consistently in future research. Identifying outcomes of importance to physicians and their patients should be prioritised, such that these may be collected within intervention studies. Reporting these consistently will allow for the effective synthesis of findings and reduce research waste. ${ }^{90}$ Integrated knowledge translation strategies involving multiple stakeholder groups (eg, physicians, patients, medical schools, physicians' associations and governing bodies, policy-makers) may help to ensure that the research is relevant and facilitates decision-making. ${ }^{91}$

\section{Strengths and limitations}

Our systematic review is the first to synthesise evidence on the effects of fatigue and insufficient sleep on physicians in independent practice. The review is timely, given recent calls for research into individual and organisational solutions for burn-out, ${ }^{20} 21$ and an increased focus on physician health. ${ }^{80}$ While we have identified a diverse body of evidence, we could not draw definitive conclusions due to methodological weaknesses (eg, 62\% of studies at high or unclear risk of bias, reliance primarily on cross-sectional designs and uncontrolled studies, subjective measurement of exposures and outcomes, small sample sizes, inclusion of predominantly male physicians within urban settings) and heterogeneous outcome measures in the included studies. Given that the 2017 update search was limited to one database, it is possible that a small number of relevant studies could have been missed. We believe that the likelihood that these might alter the conclusions of the review is low. The findings may have been influenced by publication bias and may not be generalised to all settings, given our restriction to high-income countries. Confidence in the conclusions is limited due to multiple comparisons.

\section{CONCLUSION}

The evidence synthesised in this review suggests that fatigue and insufficient sleep are associated with some detrimental physician health and well-being outcomes; the evidence for potential associations with performance and safety outcomes was mixed. Meta-analyses for patient outcomes demonstrated that in many cases, potential relationships with physician sleep deprivation remain unclear. Our overall confidence in the findings is low, owing to multiple comparisons and a body of research that is hindered by methodological weaknesses. Further methodologically robust research that includes consistent outcomes that are of interest to physicians and their patients is needed to inform strong practice recommendations and policy decisions.

Acknowledgements We thank Dr Meghan Sebastianski and Andrea Milne for assistance with screening, and Dr Allison Gates for screening, data verification and quality assessment of the French studies.
Contributors All authors contributed to the conception and design of the project. MG and AW contributed to the acquisition, analysis and interpretation of the data, and drafted the manuscript. RF contributed to acquisition of data. CSa, CSi and MPD contributed to interpretation of data and revised the manuscript for important intellectual content. All authors approved the final version of the manuscript as submitted.

Funding This research was supported by the Canadian Medical Association.

Competing interests CSi is employed by the Canadian Medical Association, who provided financial support for the research.

Patient consent Not required.

Provenance and peer review Not commissioned; externally peer reviewed.

Data sharing statement The data pertaining to this systematic review are available from the corresponding author on reasonable request.

Open access This is an open access article distributed in accordance with the Creative Commons Attribution Non Commercial (CC BY-NC 4.0) license, which permits others to distribute, remix, adapt, build upon this work non-commercially, and license their derivative works on different terms, provided the original work is properly cited, appropriate credit is given, any changes made indicated, and the use is non-commercial. See: http://creativecommons.org/licenses/by-nc/4.0/.

\section{REFERENCES}

1. Greig $P$, Snow R. Fatigue and risk: are train drivers safer than doctors? BMJ 2017;359:j5107.

2. Parker JB. The effects of fatigue on physician performance--an underestimated cause of physician impairment and increased patient risk. Can J Anaesth 1987;34:489-95.

3. Temple J. Resident duty hours around the globe: where are we now? BMC Med Educ 2014;14 Suppl 1:S8.

4. Imrie K, Frank J, The National Steering Committee on Resident Duty Hours. Fatigue, risk, \& excellence: towards a pan-Canadian consensus on resident duty hours. Ottawa, Ontario: The Royal College of Physicians and Surgeons of Canada, 2013. http://www. residentdutyhours.ca/final_report.php. (accessed 12 Jan 2018).

5. Accreditation Council for Graduate Medical Education. History of duty hours, 2017. http://www.acgme.org/What-We-Do/Accreditation/ Clinical-Experience-and-Education-formerly-Duty-Hours/History-ofDuty-Hours. (accessed 12 Jan 2018).

6. Bolster L, Rourke L. The effect of restricting residents' duty hours on patient safety, resident well-being, and resident education: an updated systematic review. J Grad Med Educ 2015;7:349-63.

7. Harris JD, Staheli G, LeClere L, et al. What effects have resident work-hour changes had on education, quality of life, and safety? A systematic review. Clin Orthop Relat Res 2015;473:1600-8.

8. Ahmed N, Devitt KS, Keshet I, et al. A systematic review of the effects of resident duty hour restrictions in surgery: impact on resident wellness, training, and patient outcomes. Ann Surg 2014;259:1041-53.

9. Peets A, Ayas NT. Restricting resident work hours: the good, the bad, and the ugly. Crit Care Med 2012;40:960-6.

10. Canadian Medical Association. CMA Policy: Management of physician fatigue. Ottawa, Canada: Canadian Medical Association, 2014.

11. Anim M, Markert RJ, Wood VC, et al. Physician practice patterns resemble ACGME duty hours. Am J Med 2009;122:587-93.

12. Transport Canada. Fatigue risk management system for the Canadian aviation industry: policies and procedures development guidelines. Ottawa, Canada: Her Majesty the Queen in Right of Canada, as represented by the Minister of Transport, 2008. https://www.tc.gc.ca/ eng/civilaviation/publications/TP14576-6042.htm. (accessed 12 Jan 2018).

13. Patterson PD, Higgins JS, Lang ES, et al. Evidence-based guidelines for fatigue risk management in ems: formulating research questions and selecting outcomes. Prehosp Emerg Care 2017;21:149-56.

14. Boudreau RA, Grieco RL, Cahoon SL, et al. The pandemic from within: two surveys of physician burnout in Canada. Can J Commun Ment Health 2006;25:71-88.

15. Kumar S. Burnout and doctors: prevalence, prevention and intervention. Health Care 2016;4:37.

16. Shanafelt TD, Boone S, Tan L, et al. Burnout and satisfaction with work-life balance among US physicians relative to the general US population. Arch Intern Med 2012;172:1377-85.

17. Brady KJS, Trockel MT, Khan CT, et al. What do we mean by physician wellness? A systematic review of its definition and measurement. Acad Psychiatry 2018;42. 
18. Fralick M, Flegel K. Physician burnout: who will protect us from ourselves? CMAJ 2014;186:731.

19. Shanafelt TD, Noseworthy JH. Executive leadership and physician well-being: nine organizational strategies to promote engagement and reduce burnout. Mayo Clin Proc 2017;92:129-46.

20. Lemaire JB, Wallace JE. Burnout among doctors. BMJ 2017;358:j3360.

21. Dyrbye LN, Trockel M, Frank E, et al. Development of a research agenda to identify evidence-based strategies to improve physician wellness and reduce burnout. Ann Intern Med 2017;166:743-4.

22. In: Higgins JPT, Green S, The Cochrane handbook for systematic reviews of interventions, version 5.1.0. London, UK: The Cochrane Collaboration, 2011. http://www.handbook.cochrane.org. (accessed 12 Jan 2018).

23. Liberati A, Altman DG, Tetzlaff J, et al. The PRISMA statement for reporting systematic reviews and meta-analyses of studies that evaluate health care interventions: explanation and elaboration. PLOS Med 2009;6:e1000100 https://doi.org/.

24. Pattani R, Wu PE, Dhalla IA. Resident duty hours in Canada: past, present and future. CMAJ 2014:186:761-5.

25. International Monetary Fund. World economic outlook: too slow for too long. Washington, DC: International Monetary Fund, 2016. http://www.imf.org/external/pubs/ft/weo/2016/01/. (accessed 12 Jan 2018).

26. Cochrane Effective Practice and Organisation of Care. Suggested risk of bias criteria for EPOC reviews. Oslo, Norway: Norwegian Knowledge Centre for the Health Services, 2016. http://epoc. cochrane.org/epoc-specific-resources-review-authors. (accessed 12 Jan 2018).

27. Wells GA, Shea B, O'Connell D, et al. The Newcastle-Ottawa Scale (NOS) for assessing the quality of nonrandomised studies in metaanalyses. Ottawa, Canada: The Ottawa Hospital Research Institute, 2014. (accessed 12 Jan 2018).

28. DerSimonian R, Laird N. Meta-analysis in clinical trials. Control Clin Trials 1986;7:177-88.

29. Higgins JP, Thompson SG, Deeks JJ, et al. Measuring inconsistency in meta-analyses. BMJ 2003;327:557-60.

30. Egger M, Davey Smith G, Schneider M, et al. Bias in meta-analysis detected by a simple, graphical test. BMJ 1997;315:629-34.

31. Govindarajan A, Urbach DR, Kumar M, et al. Outcomes of daytime procedures performed by attending surgeons after night work. $N$ Engl J Med 2015;373:845-53.

32. Chu MW, Stitt LW, Fox SA, et al. Prospective evaluation of consultant surgeon sleep deprivation and outcomes in more than 4000 consecutive cardiac surgical procedures. Arch Surg 2011:146:1080-5.

33. Furukawa TA, Barbui C, Cipriani A, et al. Imputing missing standard deviations in meta-analyses can provide accurate results. $J$ Clin Epidemiol 2006;59:7-10.

34. Amirian I, Andersen LT, Rosenberg J, et al. Laparoscopic skills and cognitive function are not affected in surgeons during a night shift. $J$ Surg Educ 2014;71:543-50.

35. Aziz A. Sources of perceived stress among American medical doctors: a cross-cultural perspective. Cross Cultural Management: An International Journal 2004;11:28-39.

36. Beaujouan L, Czernichow S, Pourriat JL, et al. [Prevalence and risk factors for substance abuse and dependence among anaesthetists: a national survey]. Ann Fr Anesth Reanim 2005;24:471-9.

37. Chang LC, Mahoney JJ, Raty SR, et al. Neurocognitive effects following an overnight call shift on faculty anesthesiologists. Acta Anaesthesiol Scand 2013;57:1051-7.

38. Chen I, Vorona R, Chiu R, et al. A survey of subjective sleepiness and consequences in attending physicians. Behav Sleep Med 2008;6:1-15.

39. Doppia MA, Estryn-Béhar M, Fry C, et al. [Burnout in French doctors: a comparative study among anaesthesiologists and other specialists in French hospitals (SESMAT study)]. Ann Fr Anesth Reanim 2011;30:782-94.

40. Dutheil F, Trousselard M, Perrier C, et al. Urinary interleukin-8 is a biomarker of stress in emergency physicians, especially with advancing age--the JOBSTRESS* randomized trial. PLOS One 2013;8:e71658

41. Ellman PI, Law MG, Tache-Leon C, et al. Sleep deprivation does not affect operative results in cardiac surgery. Ann Thorac Surg 2004:78:906-11.

42. Elovainio M, Heponiemi T, Jokela M, et al. Stressful work environment and wellbeing: What comes first? J Occup Health Psychol 2015;20:289-300.

43. Gander P, Millar M, Webster C, et al. Sleep loss and performance of anaesthesia trainees and specialists. Chronobiol Int 2008;25:1077-91.
44. Gander PH, Merry A, Millar MM, et al. Hours of work and fatiguerelated error: a survey of New Zealand anaesthetists. Anaesth Intensive Care 2000;28:178-83.

45. Gerdes J, Kahol K, Smith M, et al. Jack Barney award: the effect of fatigue on cognitive and psychomotor skills of trauma residents and attending surgeons. Am J Surg 2008;196:813-20.

46. Harbeck B, Suefke S, Haas CS, et al. No stress after 24-hour on-call shifts? J Occup Health 2015;57:438-47.

47. Heponiemi T, Puttonen S, Elovainio M. On-call work and physicians' well-being: testing the potential mediators. Occup Med 2014;64:352-7.

48. Jackson TN, Pearcy CP, Khorgami Z, et al. The physician attrition crisis: a cross-sectional survey of the risk factors for reduced job satisfaction among US Surgeons. World J Surg 2018;42:1285-92.

49. Kaneita Y, Ohida T. Association of current work and sleep situations with excessive daytime sleepiness and medical incidents among Japanese physicians. J Clin Sleep Med 2011;7:512-22.

50. Lederer W, Kopp M, Hahn O, et al. Post-duty psychomotor performance in young and senior anaesthetists. Eur $J$ Anaesthesiol 2006;23:251-6.

51. Leichtfried V, Putzer G, Perkhofer D, et al. Circadian melatonin profiles during single 24-h shifts in anesthetists. Sleep Breath 2011;15:503-12.

52. Lindfors PM, Nurmi KE, Meretoja OA, et al. On-call stress among Finnish anaesthetists. Anaesthesia 2006;61:856-66.

53. Mahmood JI, Støen Grotmol K, Tesli M, et al. Contextual factors and mental distress as possible predictors of hazardous drinking in norwegian medical doctors: a 15-year longitudinal, nationwide study. Eur Addict Res 2017;23:19-27.

54. Nishimura K, Nakamura F, Takegami M, et al. Cross-sectional survey of workload and burnout among Japanese physicians working in stroke care: the nationwide survey of acute stroke care capacity for proper designation of comprehensive stroke center in Japan (J-ASPECT) study. Circ Cardiovasc Qual Outcomes 2014;7:414-22.

55. Pit SW, Hansen V. Factors influencing early retirement intentions in Australian rural general practitioners. Occup Med 2014;64:297-304.

56. Pit SW, Hansen V. The relationship between lifestyle, occupational health, and work-related factors with presenteeism amongst general practitioners. Arch Environ Occup Health 2016;71:49-56.

57. Roberts DL, Shanafelt TD, Dyrbye LN, et al. A national comparison of burnout and work-life balance among internal medicine hospitalists and outpatient general internists. J Hosp Med 2014;9:176-81.

58. Rothschild JM, Keohane CA, Rogers S, et al. Risks of complications by attending physicians after performing nighttime procedures. JAMA 2009;302:1565-72.

59. Saadat $\mathrm{H}$, Bissonnette $\mathrm{B}$, Tumin $\mathrm{D}$, et al. Effects of partial sleep deprivation on reaction time in anesthesiologists. Paediatr Anaesth 2017;27:358-62.

60. Saadat H, Bissonnette B, Tumin D, et al. Time to talk about workhour impact on anesthesiologists: The effects of sleep deprivation on Profile of Mood States and cognitive tasks. Paediatr Anaesth 2016;26:66-71.

61. Sanches I, Teixeira F, dos Santos JM, et al. Effects of acute sleep deprivation resulting from night shift work on young doctors. Acta Med Port 2015;28:457-62.

62. Sargent MC, Sotile W, Sotile MO, et al. Quality of life during orthopaedic training and academic practice. Part 1: orthopaedic surgery residents and faculty. J Bone Joint Surg Am 2009;91:2395-405.

63. Schieman C, MacLean AR, Buie WD, et al. Does surgeon fatigue influence outcomes after anterior resection for rectal cancer? Am J Surg 2008;195:684-8.

64. Sende J, Jbeili C, Schvahn S, et al. Stress factors and stress consequences among emergency physicians: vocational survey. Annales Francaises de Médecine d'Urgence 2012;2:224-31.

65. Sexton JB, Thomas EJ, Helmreich RL, et al. Error, stress, and teamwork in medicine and aviation: cross sectional surveys. BMJ 2000;320:745-9.

66. Shanafelt TD, Balch CM, Bechamps G, et al. Burnout and medical errors among American surgeons. Ann Surg 2010;251:995-1000.

67. Shanafelt TD, Novotny P, Johnson ME, et al. The well-being and personal wellness promotion strategies of medical oncologists in the North Central Cancer Treatment Group. Oncology 2005;68:23-32.

68. Shanafelt TD, Raymond M, Kosty M, et al. Satisfaction with work-life balance and the career and retirement plans of US oncologists. $J$ Clin Oncol 2014;32:1127-35.

69. Shirom A, Nirel N, Vinokur AD, et al. Overload, autonomy, and burnout as predictors of physicians' quality of care. $J$ Occup Health Psychol 2006;11:328-42. 
70. Shirom A, Nirel N, Vinokur AD. Work hours and caseload as predictors of physician burnout: the mediating effects by perceived workload and by autonomy. Appl Psychol 2010;59:539-65.

71. Smith F, Goldacre MJ, Lambert TW. Adverse effects on health and wellbeing of working as a doctor: views of the UK medical graduates of 1974 and 1977 surveyed in 2014. J R Soc Med 2017;110:198-207.

72. Starmer AJ, Frintner MP, Freed GL, et al. Work-life balance, burnout, and satisfaction of early career pediatricians. Pediatrics 2016;137:e20153183.

73. Tanti A, Camilleri M, Borg AA, et al. Opinions of Maltese doctors and pharmacists on medication errors. Int J Risk Saf Med 2017;29(12):81-99.

74. Tokuda Y, Hayano K, Ozaki M, et al. The interrelationships between working conditions, job satisfaction, burnout and mental health among hospital physicians in Japan: a path analysis. Ind Health 2009;47:166-72.

75. Uchal M, Tjugum J, Martinsen E, et al. The impact of sleep deprivation on product quality and procedure effectiveness in a laparoscopic physical simulator: a randomized controlled trial. Am J Surg 2005;189:753-7.

76. Vela-Bueno A, Moreno-Jiménez B, Rodríguez-Muñoz A, et al. Insomnia and sleep quality among primary care physicians with low and high burnout levels. J Psychosom Res 2008;64:435-42.

77. Vinden C, Nash DM, Rangrej J, et al. Complications of daytime elective laparoscopic cholecystectomies performed by surgeons who operated the night before. Obstet Gynecol Surv 2014;69:71-3.

78. Wada K, Yoshikawa T, Goto T, et al. National survey of the association of depressive symptoms with the number of off duty and on-call, and sleep hours among physicians working in Japanese hospitals: a cross sectional study. BMC Public Health 2010;10:127.

79. Sturm $L$, Dawson D, Vaughan $R$, et al. Effects of fatigue on surgeon performance and surgical outcomes: a systematic review. ANZ J Surg 2011;81(7-8):502-9.
80. Wallace JE, Lemaire JB, Ghali WA. Physician wellness: a missing quality indicator. Lancet 2009;374:1714-21.

81. Canadian Medical Association. CMA policy: physician health Ottawa, Canada: Canadian Medical Assocation, 2017. http:// policybase.cma.ca/dbtw-wpd/Policypdf/PD18-01.pdf. (accessed 12 Jan 2018).

82. Shanafelt T, Goh J, Sinsky C. The business case for investing in physician well-being. JAMA Intern Med 2017;177:1826-205.

83. Gaba DM, Howard SK. Patient safety: fatigue among clinicians and the safety of patients. N Engl J Med 2002;347:1249-55.

84. Ward S, Outram S. Medicine: in need of culture change. Intern Med $J$ 2016;46:112-6.

85. Panagioti M, Panagopoulou E, Bower P, et al. Controlled interventions to educe urnout in hysicians: A Systematic Review and Meta-analysis. JAMA Intern Med 2017;177:195-205.

86. American Medical Association. STEPSforwardTM. Chicago, IL: American Medical Assocation, 2017. https://www. stepsforward.org/modules/joy-in-medicine. (accessed 12 Jan 2018).

87. Jackson C. The Chalder Fatigue Scale (CFQ 11). Occup Med 2015;65:86

88. Chalder T, Berelowitz G, Pawlikowska T, et al. Development of a fatigue scale. J Psychosom Res 1993;37:147-53.

89. Smets EM, Garssen B, Bonke B, et al. The Multidimensional Fatigue Inventory (MFI) psychometric qualities of an instrument to assess fatigue. J Psychosom Res 1995;39:315-25.

90. Ioannidis JP, Greenland S, Hlatky MA, et al. Increasing value and reducing waste in research design, conduct, and analysis. Lancet 2014;383:166-75.

91. Gagliardi AR, Berta W, Kothari A, et al, Integrated knowledge translation (IKT) in health care: a scoping review. Implement Sci 2016;11:38 https://doi.org/10.1186/s13012-016-0399-1. 